\title{
Dissecting Aneurysm of the Anterior Cerebral Artery with Severe Subarachnoid Hemorrhage during Treatment for Cerebral Infarction
}

\author{
Shoko M. YAMADA, Makoto NAKANE, Makoto AOKI and Hitoshi NAKAYAMA
}

\begin{abstract}
Intracranial dissecting aneurysms cause ischemia, but anticoagulation or antiplatelet agents are administered to most ischemic patients without angiographical investigation. A 55-year-old woman succumbed to a subarachnoid hemorrhage (SAH) during antiplatelet therapy for ischemia caused by a dissecting aneurysm at the anterior cerebral artery, which was identified by conventional angiography on day 11 after admission. The authors emphasize that all dissecting aneurysms manifesting ischemic attack can cause hemorrhage. Therefore, emergency angiography is recommended for patients with ischemia complaining of a headache. If dissection is identified, it may be better to regulate the blood pressure of the patient strictly without anticoagulation or antiplatelet therapy.
\end{abstract}

(Internal Medicine 42: 433-435, 2003)

Key words: SAH, ACA, dissection, aneurysm

\section{Introduction}

Non-traumatic dissecting aneurysms occur in both the intra- and extra-cranial arteries. The most common aneurysm sites are the vertebro-basilar artery (VA-BA), the middle cerebral artery (MCA), and the internal carotid artery (ICA) $(1,2)$. Such aneurysms are classified into two groups according to their first manifestation: hemorrhagic and nonhemorrhagic. The former is characterized by subarachnoid hemorrhage (SAH) and intracerebral hemorrhage (ICH), and the latter by ischemic attack, neurological deficits caused by the mass effect of the aneurysm, and incidental finding only by a headache. The present report focuses on the treatment of dissecting aneurysms that manifest ischemia, with reference to our experience of a patient who died of SAH during treatment for an ischemic attack caused by a dissecting aneurysm of the anterior cerebral artery (ACA).

\section{Case Report}

A 55-year-old woman visited our clinic complaining of sudden onset of confusion with a mild headache in the frontal region. Her consciousness level was 13 on the Glasgow Coma Scale (GCS) (E4V4M5), and no neurological deficits were detected except for disorientation. The patient had a history of hypertension, and had been treated with nifedipine (Adalat L $20 \mathrm{mg}$ twice daily) for about 2 years. On admission her blood pressure (BP) was 167/95, and pulse rate was 84 without arrhythmia. The patient had no history of diabetes mellitus, myocardial infarction, or cerebral infarction. A computed tomography (CT) scan demonstrated ischemic changes in the left frontal lobe, where the blood is supplied from the distal portion of the left ACA (Fig. 1). The patient was admitted to our hospital and treated with the antiplatelet agent, sodium ozagrel (Xanbon $80 \mathrm{mg} /$ day). On the second day of admission, magnetic resonance angiography (MRA) and magnetic resonance imaging (MRI) were performed, but no definite arterial abnormality was identified (Fig. 2A and 2B). Her systolic BP was kept below $160 \mathrm{mmHg}$ by continuous venous infusion of nicardipine hydrochloride $(2 \mu \mathrm{g} / \mathrm{kg} /$ min) and the sodium ozagrel antiplatelet therapy was then continued, since we concluded that the infarction had been caused by atheromatous thrombosis of a non-specific artery. However, a diagnosis of dissecting aneurysm was confirmed by conventional angiography conducted on day 11 of admission. This revealed dilatation at the A2 portion of the left ACA (from the anterior communicating artery to a point just proximal to the division of the pericallosal and callosomarginal arteries) (Fig. 3 white arrow), and no other abnomalities were identified in any portion of the intracranial arteries. On the day following the angiography, the patient's conscious-

From the Department of Neurosurgery, Teikyo University School of Medicine, Mizonokuchi Hospital, Kawasaki

Received for publication October 10, 2002; Accepted for publication February 17, 2003

Reprint requests should be addressed to Dr. Shoko M. Yamada, Department of Neurosurgery, Teikyo University School of Medicine, Mizonokuchi Hospital, 3-8-3 Mizonokuchi, Takatsu-ku, Kawasaki 213-8507 


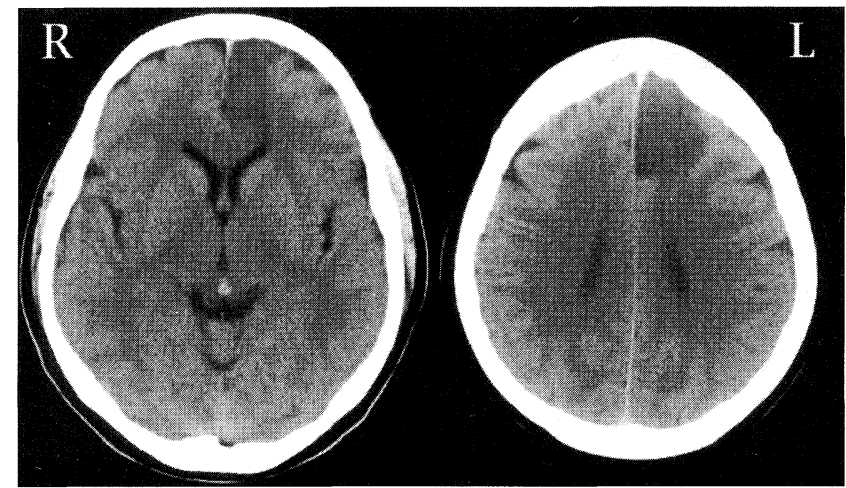

Figure 1. Plain computed tomography scan shows a lowdensity area in the left frontal lobe, suggesting cerebral infarction at the distal portion of the left anterior cerebral artery.

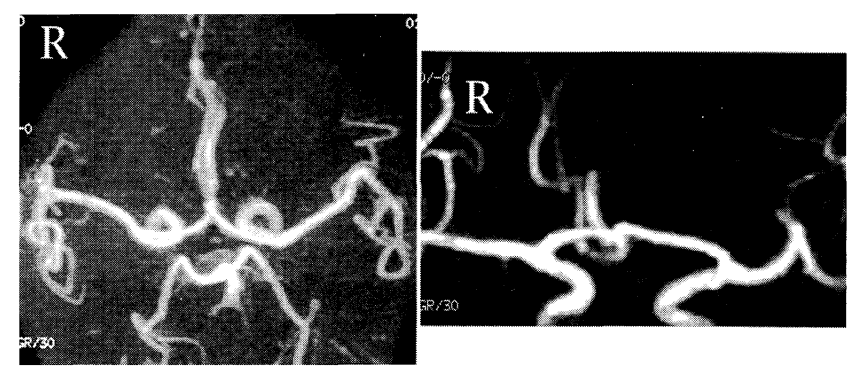

A

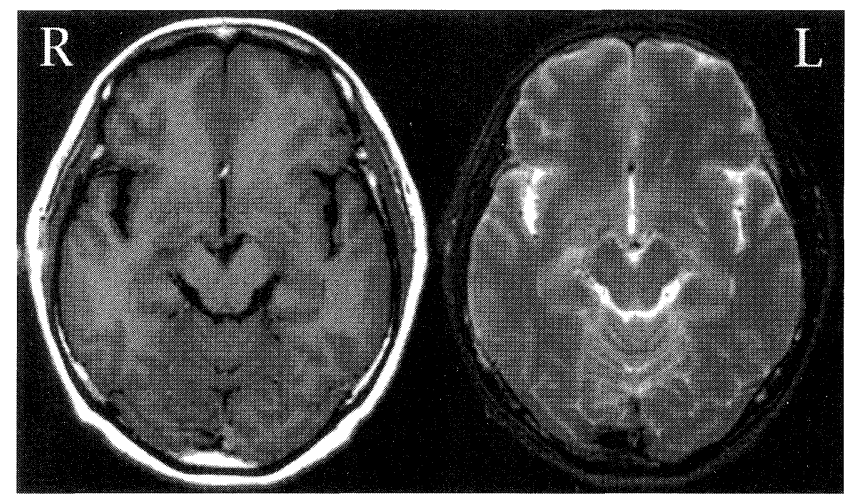

B

Figure 2. A) No obvious intracranial arterial abnormalities, such as occlusion or dissection, are identified at the left anterior cerebral artery by magnetic resonance angiography (MRA). (Signa 1.5 T, 3D-time of flight technique, repetition time $(\mathrm{TR})=$ $69 \mathrm{msec}$, echo time (TE) $=3.1 \mathrm{msec}$, flip angle of $30,0.7 \mathrm{~mm}$ thickness). B) T1-weighted magnetic resonance imaging (MRI) (Signa 1.5 T, spin-echo, TR=300, TE=10) demonstrates a spotty high-intensity lesion at the anterior cerebral artery suggesting dissection with a blood clot. However, it is difficult to diagnose a dissecting aneurysm by T2-weighted MRI (Signa, 1.5 T, fast spin-echo, $T R=6,000, T E=104)$.

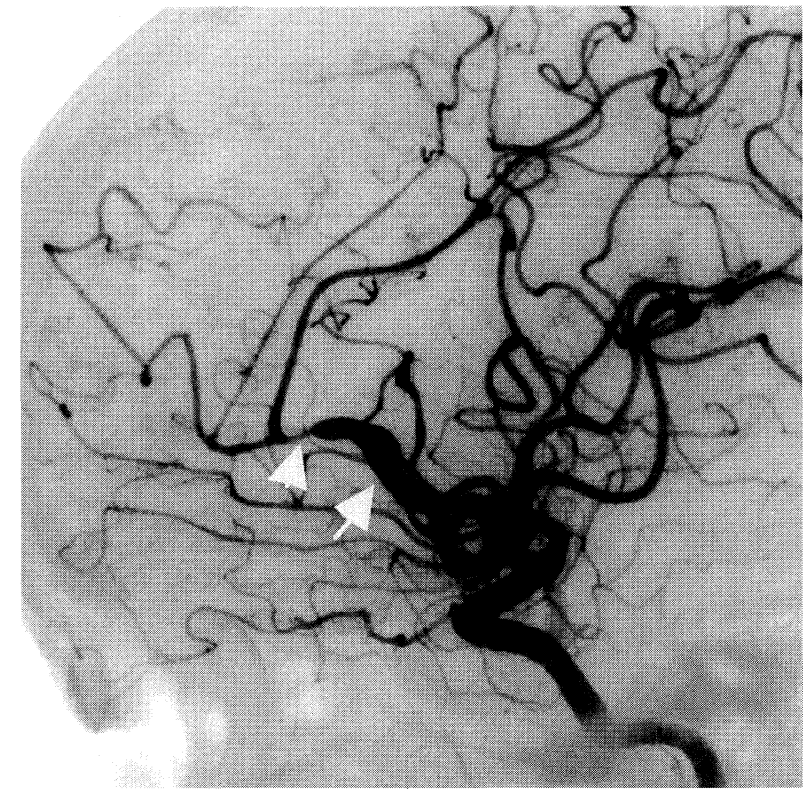

Figure 3. Conventional angiography reveals unusual dilatation of the left anterior cerebral artery (white arrow), with stenosis just distal to the end of the dilatation (white arrow head). Cerebral blood flow in the left distal anterior cerebral artery, particularly the A3 to A4 portion, is well preserved.

ness level deteriorated suddenly to GCS level 4 (E1V1M2), and a CT scan revealed severe SAH with diffuse brain swelling, suggesting impending herniation (Fig. 4). Emergency surgery to trap the left A2 portion with external decompression was performed, but the patient succumbed to the SAH three days after the operation.

\section{Discussion}

Non-traumatic dissecting aneurysms at the ACA are extremely rare compared with those at the VA-BA, MCA, and ICA $(1,2)$. It has been reported that dissecting aneurysms of the posterior circulation often cause $\mathrm{SAH}$, whereas those of the anterior circulation induce ischemia $(2,3)$. This observation is supported by the fact that the reported incidence of $\mathrm{SAH}$ is $87.5 \%$ in VA dissecting aneurysms (4), but only $14 \%$ in aneurysms of the anterior circulation (5).

Unlike hemorrhagic ictus cases, emergency angiography is not usually performed in ischemic patients unless MCA or ICA occlusion is suspected, and early administration of antiplatelet or anticoagulation agents to patients with ischemic stroke is a common treatment. Although ischemia may be aggravated by conventional angiography, especially in the acute phase, emergency angiography should have been achieved in this patient, because the ischemic change was located in an atypical area of the brain and she complained of headache, which is the most common symptom even in patients with cerebral infarction caused by an intracranial dissecting aneurysm. It is very difficult to predict that ischemia 


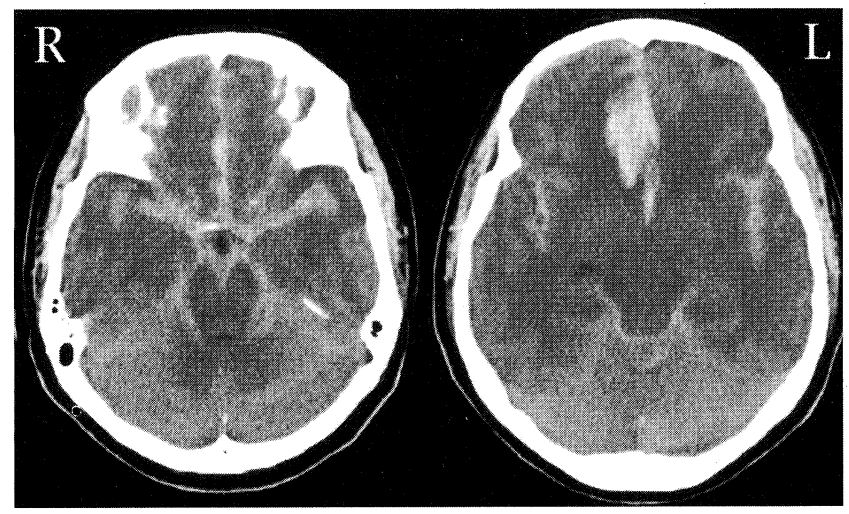

Only Honda et al have reported a case of SAH caused by a left A1A2 dissecting aneurysm one month after an ischemic attack caused by the aneurysm, but they did not mention whether they treated the patient with antiplatelet or anticoagulation agents (10). Yamaura et al reported that only 3.4\% (4 of 118 cases) of dissecting aneurysms with ischemic manifestations were complicated by SAH (11). Despite the low incidence of SAH during the treatment of dissecting aneurysm, it is noteworthy that six of the eight patients reported by Inagaki et al and three of the four reported by Yamaura et al died. Therefore, it is suggested that the prognosis of patients with ischemia caused by a dissecting aneurysm becomes very poor if complicated by SAH. It has been reported that the outcome in patients with ischemia caused

Figure 4. Severe subarachnoid hemorrhage with hematoma at the right rectal gyrus is revealed by computed tomography scan. Severe brain swelling is visible as a diffuse low-density area in both hemispheres with a narrow space around the brainstem, implying impending herniation of the brain.

is caused by a dissection of the intracranial artery using CT scan alone, and MRA is considered to be more useful in this respect (6). However, not all intracranial dissecting aneurysms can be identified merely by MRA, and it was very difficult in the present case to detect the arterial abnormality by MRA or MRI (Fig. 2A and 2B).

Pathological investigations have proved that subintimal types of dissecting aneurysm are manifested as ischemia whereas subadventitial types cause SAH (3). In an angiographic study of dissecting aneurysms, Ohkuma et al reported that arterial stenosis with dilatation occurred predominantly in SAH cases, whereas stenosis without dilatation arose mainly in ischemic cases (7). However, the authors consider that all intracranial dissecting aneurysms can cause hemorrhage because any type of aneurysm has the common feature of weakened vessel walls. We intended to perform trapping of the left A2 portion with anastomosis of the left $\mathrm{A} 3$ to the right $\mathrm{A} 3$ portion after completion of the two-week antiplatelet treatment for cerebral infarction.

It was difficult to conclude what induced the SAH in the present patient. Angiography might have provoked the SAH, but all the patient's vital signs were stable during and after the examination, and the dissection was located in the peripheral ACA where the injection pressure of contrast medium would have had only an indirect effect. The antiplatelet therapy could have induced the severe SAH in this case, and in fact, Lagares et al reported a patient with acute myocardial infarction who died of severe SAH from a saccular aneurysm of the anterior communicating artery 8 hours after administration of tissue plasminogen activator (TPA). They concluded that the thrombolytic therapy might have induced the hemorrhage (8). Inagaki et al reviewed eight cases of dissecting aneurysm in which SAH occurred during treatment for ischemia (9), but all of these were VA dissecting aneurysms. by a dissecting aneurysm did not differ significantly between groups of patients treated with or without antiplatelet or anticoagulation agents (11). Therefore, we consider that patients with a dissecting aneurysm producing ischemia are best managed by strict control of their blood pressure without the use of antiplatelet or anticoagulation agents, to avoid the risk of inducing SAH. In some cases, surgical treatment should be instituted to prevent sequential SAH considering the location of the dissection and the shape of the aneurysm.

\section{References}

1) Kidooka M, Okada T, Sonobe M, Nakazawa T, Handa J. Dissecting aneurysm of the anterior cerebral artery: report of two cases. Surg Neurol 39: $53-57,1993$.

2) Wakabayashi $Y$, Nakano T, Isono M, Shimomura T, Hori S. Dissecting aneurysm of the anterior cerebral artery requiring surgical treatment: case report. Neurol Med Chir (Tokyo) 40: 624-627, 2000.

3) Yonas H, Agamanolis D, Takaoka Y, White RJ. Dissecting intracranial aneurysms. Surg Neurol 8: 407-415, 1977.

4) Yamaura A, Watanabe $Y$, Saeki N. Dissecting aneurysms of the intracranial vertebral artery. J Neurosurg 72: 183-188, 1990.

5) Tanaka K, Waga S, Kojima T, Kubo Y, Shimizu T, Niwa S. Nontraumatic dissecting aneurysms of the intracranial vertebral artery: report of six cases. Acta Neurochir (Wien) 100: 62-66, 1989.

6) Lanzino G, Kaptain G, Kallmes DF, Dix JE, Kassell NF. Intracranial dissecting aneurysm causing subarachnoid hemorrhage: the role of computerized tomographic angiography and magnetic resonance angiography. Surg Neurol 48: 477-481, 1997.

7) Ohkuma H, Suzuki S, Ogane K. Dissecting aneurysms of intracranial carotid circulation. Stroke 33: 941-947, 2002.

8) Lagares A, Gomez PA, Lobato RD, Alen JF, Campollo J, Benito-Leon J. Cerebral aneurysm rupture after r-TPA thrombolysis for acute myocardial infarction. Surg Neurol 52: 623-626, 1999.

9) Inagaki $T$, Saito K, Hirano A, Kato T, Irie S, Murakami T. Vertebral arterial dissection with subarachnoid hemorrhage after ischemic onset. No Shinkei Geka 28: 997-1002, 2000 (in Japanese, Abstract in English).

10) Honda N, Yuge T, Miyagi J, Shigemori M. A surgical case of dissecting aneurysm of the anterior cerebral artery. Jpn J Neurosurg (Tokyo) 6: 634-638, 1997 (in Japanese, Abstract in English).

11) Yamaura A, Yoshimoto T, Hashimoto N, Ono J. Nationwide study of nontraumatic intracranial arterial dissection: treatment and its result. Surg Cereb Stroke (Jpn) 26: 87-95, 1998 (in Japanese, Abstract in English). 